\title{
PREDICTION OF NET BEDLOAD TRANSPORT RATES OBTAINED IN OSCILLATING WATER TUNNELS AND APPLICABILITY TO REAL SURF ZONE WAVES
}

\author{
David Gonzalez-Rodriguez ${ }^{1}$ and Ole Secher Madsen ${ }^{2}$
}

\begin{abstract}
Experimental studies of sediment transport rates due to nearshore waves are often conducted in oscillating water tunnels (OWTs). In an OWT, the oscillatory motion produced by the piston propagates almost instantaneously along the entire tunnel. Consequently, unlike the wave motion in the sea or in a wave flume, flow in an OWT is uniform along the tunnel, and second-order wave propagation effects (such as Longuet-Higgins's streaming) are absent. The effect of these hydrodynamic differences between OWT and sea waves on sediment transport rates has generally been neglected. In this paper we present a simple, practical formulation to evaluate bed shear stresses and bedload transport rates due to asymmetric and skewed waves plus a current in an OWT, based on fitting the exact results of a rigorous, analytical model of the OWT wave-current boundary layer. By then accounting for real wave effects we find that wave propagation significantly affects the predicted period-averaged net sediment transport rates. Such real wave effects can therefore not be neglected when comparing nearshore transport models with OWT data.
\end{abstract}

Keywords: nearshore sediment transport; bedload; oscillating water tunnel; wave-current boundary layer; wave asymmetry; wave skewness

\section{INTRODUCTION}

Cross-shore sediment transport in the nearshore region is due to the simultaneous effect of waves and currents. Nearshore waves are both asymmetric (forward-leaning in shape) and skewed (with peaked, narrow crests and wide, flat troughs). Most existing nearshore sediment transport models are either highly empirical or require intensive numerical computation. Moreover, while most experimental nearshore sediment transport studies have been conducted in oscillating water tunnels (OWTs), most existing models disregard hydrodynamic differences between OWTs (where waves do not propagate) and the sea. As discussed in this paper, these hydrodynamic differences have a significant effect on sediment transport.

Recently we have developed an analytical sediment transport model due to nearshore waves and currents. We first proposed a simple conceptual model capable of predicting bedload transport due to asymmetric and skewed pure waves (Gonzalez-Rodriguez and Madsen 2007), which we later extended to the case of combined waves and currents (Gonzalez-Rodriguez and Madsen 2008). In order to correctly predict net sediment transport rates, this earlier model required an inconsistent choice of the effective sheet flow roughness, which was taken equal to the sediment diameter for the case of pure waves and equal to the total, mobile-bed roughness for the case of combined sinusoidal waves plus a current. To overcome this inconsistency, we developed an analytical model of the wave-current boundary layer (Gonzalez-Rodriguez and Madsen 2010, see also Gonzalez-Rodriguez 2009 for details; hereafter this model is referred to as GRM10). The model was developed for the non-propagating motion in an OWT, so that its results could consistently be compared with OWT experimental measurements. Using a space- and time-dependent eddy viscosity, we obtained explicit analytical expressions for the bed shear stress, which can readily be used to compute bedload transport. Good agreement with bedload measurements was consistently obtained for pure waves and for combined waves and currents when the effective sheet flow roughness was parameterized by the total, mobile-bed roughness, thus overcoming the inconsistency of our earlier model. However, the analytical expressions in GRM10 involve Kelvin functions and complex numbers, making its numerical evaluation slightly cumbersome.

In this paper we first summarize the key steps in deriving GRM10. Then we present a new, simplified formulation for the bed shear stresses predicted by GRM10, intended to facilitate the model's application. In this simplified formulation, the bed shear stress is simply expressed in terms of friction factors and phase shifts, which are fits to the exact GRM10 analytical expressions. We show that application of these fitted expressions results in similarly accurate predictions of the bedload transport rates as the original GRM10.

\footnotetext{
${ }^{1}$ Formerly at: Department of Civil and Environmental Engineering, Massachusetts Institute of Technology, Cambridge, MA 02139, USA. Currently at: Institut Curie, Paris 75005, France. E-mail: davidgr@alum.mit.edu ${ }^{2}$ Department of Civil and Environmental Engineering, Massachusetts Institute of Technology, Cambridge, MA 02139, USA. E-mail: osm@mit.edu
} 
The excellent predictive ability of GRM10 for non-propagating (OWT) wave conditions validates our approach to computation of sediment transport. The next step will be to extend this formulation to the case of propagating waves, in the way outlined by Gonzalez-Rodriguez (2009). At the end of this paper, we use Trowbridge and Madsen's (1984a,b) analytical model to obtain a preliminary estimation of the effect of wave propagation on bedload sediment transport rates, which we find to be significant. We thus conclude that hydrodynamic differences between OWTs and sea waves cannot be neglected when using OWT measurements in the development of nearshore sediment transport models.

\section{ANALYTICAL MODEL OF THE OWT WAVE-CURRENT BOUNDARY LAYER}

Here we summarize the key aspects of GRM10; the detailed derivation of the model is presented in Gonzalez-Rodriguez and Madsen (2010). GRM10 consists of an approximate analytical solution of the wave boundary layer equation for a non-propagating oscillatory motion. Since waves do not propagate, the nonlinear advective terms are absent from the boundary layer equation, which reads:

$$
\frac{\partial u}{\partial t}=-\frac{1}{\rho} \frac{\partial p}{\partial x}+\frac{1}{\rho} \frac{\partial \tau_{z x}}{\partial x}
$$

where $u$ is the horizontal velocity, $p$ is the pressure, $\rho$ is the fluid density, and $\tau_{z x}$ is the dominant shear stress component, given by

$$
\tau_{z x}=\rho v_{t} \frac{\partial u}{\partial z}
$$

$v_{t}(z, t)$ is the eddy viscosity, which is assumed isotropic but to depend on both the distance from the boundary and on time, according to

$$
v_{t}(z, t)=\bar{v}(z)\left(\frac{1}{2}+\frac{a^{(1)}}{2} e^{i \omega t}+\frac{a^{(2)}}{2} e^{i 2 \omega t}+\mathrm{cc}\right)
$$

where $\omega=2 \pi / T$ is the radian frequency. Based on law-of-the-wall arguments in the wave boundary layer (of thickness $\delta_{w}$ ) and in the current boundary layer (of thickness $\delta_{c}$ ), and following previous turbulent flow studies, the $z$-dependence of the eddy viscosity is defined by

$$
\bar{v}(z)= \begin{cases}\kappa u_{*_{w}} z & 0 \leq z \leq \delta_{I} \\ \kappa u_{*} \delta_{I} & \delta_{I}<z \leq \delta_{J} \\ \kappa u_{*} z & \delta_{J}<z \leq \delta_{K} \\ \kappa u_{* c} \delta_{L} & \delta_{K}<z,\end{cases}
$$

where $\delta_{I} \equiv \delta_{w} / 6, \delta_{L} \equiv \delta_{c} / 6, \delta_{w c} \equiv \mathrm{u}{ }_{\mathrm{wc}} \delta_{I} / \mathrm{u}_{*_{\mathrm{c}}}$,

$$
\delta_{\mathrm{J}} \equiv \begin{cases}\min \left(\delta_{w}, \delta_{w c}\right) & u_{*_{w c}} \delta_{I}<u_{*_{c}} \delta_{L} \\ \delta_{w} & u_{*_{w c}} \delta_{I} \geq u_{*_{c}} \delta_{L},\end{cases}
$$

and $\delta_{K} \equiv \max \left(\delta_{J}, \delta_{L}\right)$. The current boundary layer thickness $\delta_{c}$ is taken as the height of the OWT section. The wave boundary layer thickness is given by $\delta_{W}=A l$, where the boundary layer length scale is $l \equiv \kappa u_{{ }_{w c}} / \omega$. We define the boundary layer thickness as the distance from the bottom where the velocity amplitude differs by $1 \%$ from the free-stream velocity amplitude, from which we obtain the following fitting for the coefficient $A$ :

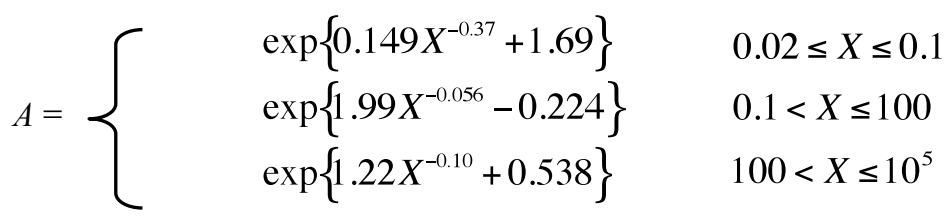

This expression depends on the relative roughness, $X=u_{b m, 1} /\left(k_{n} \omega\right)$, where $k_{n}$ is the effective roughness of the bottom. As discussed by Gonzalez-Rodriguez and Madsen (2010), for sheet flow this effective roughness is equal to the total, mobile-bed roughness, given by Herrmann and Madsen's (2007) formula: 


$$
k_{n}=\left[4.5\left(\psi-\psi_{c r}\right)+1.7\right] D_{n},
$$

where $\psi$ and $\psi_{c r}$ are the Shields parameter and its critical value for initiation of motion, respectively, and $D_{n} \sim 1.1 D_{50}$ is the nominal diameter. It is noted that the roughness, which is needed to compute the bed shear stress, depends in turn on the bed shear stress through the Shields parameter. Thus, a few iterations are required to calculate the roughness.

The time dependence of the eddy viscosity is approximated by a mean, first and second harmonics, the amplitudes of which $\left(a^{(1)}\right.$ and $\left.a^{(2)}\right)$ are determined by assuming that the instantaneous eddy viscosity scales with the instantaneous bed shear stress, i.e.,

$$
v_{t}(z, t)=\kappa z\left|\tau_{b} / \rho\right|^{1 / 2},
$$

where $\tau_{b}$ is the bed shear stress and $\kappa \sim 0.4$ is von Kármán's constant. The free-stream velocity is approximated by its two first Fourier harmonics, i.e.,

$$
u_{b}(t)=\frac{U_{\infty}^{(1)}}{2} e^{i \omega t}+\frac{U_{\infty}^{(2)}}{2} e^{i 2 \omega t}+\mathrm{cc} .
$$

This expression can approximate a realistic range of asymmetric and skewed waves by an appropriate choice of the phase difference between the two harmonics. Given a real near-bed wave velocity series, the best two-component approximation (9) that should be used as the model input is obtained by taking $U_{\infty}^{(1)}$ and $U_{\infty}^{(2)}$ equal to the complex amplitudes of the two first harmonics of the Fourier transform of the velocity series.

In order to analytically solve equation (1), both the second-harmonic wave velocity and the current velocity are assumed small compared to the first harmonic velocity, i.e.,

$$
\lambda=\frac{\left|U_{\infty}^{(2)}\right|}{\left|U_{\infty}^{(1)}\right|} \sim \frac{u_{*_{c}}^{2}}{u_{*_{w}}^{2}} \ll<,
$$

where $u_{*_{c}}$ is the current shear velocity. The equation is then solved by series expansion in terms of the small parameter, $\lambda$. After long algebra, explicit expressions are obtained for the velocities and shear stresses up to order $\lambda$. Most notably, the bed shear stress is expressed as a sum of a mean plus a first, second, and third harmonic.

\section{APPROXIMATE EXPRESSIONS FOR THE BED SHEAR STRESS}

Here we present approximate fittings of the bed shear stress obtained by GRM10. For details on the derivation of these fittings the reader is referred to Gonzalez-Rodriguez (2009). The near-bed velocity (9) can be rewritten without the use of complex numbers as

$$
u_{b}(t)=u_{b m, 1} \cos (\omega t)+u_{b m, 2} \cos [2(\omega t+\phi)] .
$$

Here, $u_{b m, 1}$ and $u_{b m, 2}$ are the real amplitudes of the first and second harmonics, respectively, while the complex amplitudes (which include phase information) were denoted above by $U_{\infty}^{(1)}$ and $U_{\infty}^{(2)}$. The wave bed shear stress is written as

$$
\begin{aligned}
& \tau_{b w}(t)=\tau_{b m, 1} \cos \left(\omega t+\varphi_{1}\right)+\tau_{b m, 2 \alpha} \cos \left[2\left(\omega t+\varphi_{2 \alpha}\right)\right]+\tau_{b m, 2 \beta} \cos \left[2\left(\omega t+\varphi_{2 \beta}\right)\right] \\
& +\tau_{b m, 3} \cos \left[3\left(\omega t+\varphi_{3}\right)\right] .
\end{aligned}
$$

In this expression the second harmonic is written as the sum of two terms, as required to fit the exact analytical solution. It is noted that the wave-induced mean shear stress is neglected in this approximate expression. By comparing bedload transport predictions for pure waves using the exact analytical solution with and without the mean stress, we conclude that the effect of the mean stress on the net bedload transport rate is typically smaller than $20 \%$.

Next, the amplitudes of the wave bed shear stress components are written in terms of friction factors as

$$
\tau_{b m, 1}=\frac{1}{2} \rho f_{1} u_{b m, 1}^{2}
$$




$$
\begin{aligned}
& \tau_{b m, 2 \alpha}=\frac{1}{2} \rho f_{2 \alpha} u_{b m, 1} u_{b m, 2} \\
& \tau_{b m, 2 \beta}=\frac{1}{2} \rho f_{2 \beta} u_{b m, 1} u_{b m, 2} \\
& \tau_{b m, 3}=\frac{1}{2} \rho f_{3} u_{b m, 3}^{2} .
\end{aligned}
$$

The friction factors are given by the following fittings:

$$
\begin{aligned}
& f_{1}= \begin{cases}\exp \left\{17.6 X^{-0.05}-20.4\right\} & 0.02 \leq X \leq 0.1 \\
\exp \left\{10.17 X^{-0.05}-12.1\right\} & 0.1<X \leq 100 \\
\exp \left\{5.84 X^{-0.11}-7.54\right\} & 100<X \leq 10^{5}\end{cases} \\
& f_{2 \alpha}=\left\{\begin{array}{lll} 
& \exp \left\{-21.6 X^{0.05}+19.3\right\} & 0.02 \leq X \leq 0.1 \\
& \exp \left\{9.78 X^{-0.06}-11.2\right\} & 0.1<X \leq 100 \\
\exp \left\{6.05 X^{-0.11}-7.42\right\} & 100<X \leq 10^{5}
\end{array}\right. \\
& f_{2 \beta}= \begin{cases}\exp \left\{18.7 X^{-0.05}-23.2\right\} & 0.02 \leq X \leq 0.1 \\
\exp \left\{8.25 X^{-0.07}-11.9\right\} & 0.1<X \leq 100 \\
\exp \left\{5.50 X^{-0.11}-9.19\right\} & 100<X \leq 10^{5}\end{cases} \\
& f_{3}= \begin{cases}\exp \left\{-25.8 X^{0.04}+20.9\right\} & 0.02 \leq X \leq 0.1 \\
\exp \left\{10.23 X^{-0.05}-14.1\right\} & 0.1<X \leq 100 \\
\exp \left\{5.60 X^{-0.11}-9.32\right\} & 100<X \leq 10^{5}\end{cases}
\end{aligned}
$$

The phase shifts for each of the wave bed shear stress components are given by

$$
\begin{gathered}
\varphi_{1}(\mathrm{rad})=\left\{\begin{array}{cl}
0.660 & 0.02 \leq X \leq 0.045 \\
-0.168\left(\log _{10} X\right)^{2}-0.105 \log _{10} X+0.825 & 0.045<X \leq 2 \\
0.0202\left(\log _{10} X\right)^{2}-0.228 \log _{10} X+0.846 & 2<X \leq 10^{5}
\end{array}\right. \\
\varphi_{2 \alpha}(\mathrm{rad})=4.8 \cdot 10^{-3}\left(\log _{10} X\right)^{2}-0.074 \log _{10} X+0.34+\phi \\
\varphi_{2 \beta}(\mathrm{rad})=-6.3 \cdot 10^{-3}\left(\log _{10} X\right)^{3}+0.096\left(\log _{10} X\right)^{2}-0.53 \log _{10} X-\phi \\
\varphi_{3}(\mathrm{rad})=-7.19 \cdot 10^{-3}\left(\log _{10} X\right)^{3}+0.0967\left(\log _{10} X\right)^{2}-0.474 \log _{10} X+1.03,
\end{gathered}
$$

where (19), (20), and (21) are valid for $0.02 \leq \mathrm{X} \leq 10^{5}$. Finally, the wave-current shear velocity is computed as

$$
u_{*_{w c}}=\overline{\left|\frac{\tau_{b}}{\rho}\right|^{1 / 2}}=u_{b m, 1} \sqrt{\frac{1}{2} \rho f_{a v e}},
$$

where the fitted expression for the average friction factor is

$$
f_{\text {ave }}=\left\{\begin{array}{lll} 
& \exp \left\{-53.3 X^{0.02}+49.7\right\} & 0.02 \leq X \leq 0.1 \\
& \exp \left\{7.78 X^{-0.07}-10.30\right\} & 0.1<X \leq 100 \\
& \exp \left\{5.87 X^{-0.11}-8.20\right\} & 100<X \leq 10^{5}
\end{array}\right.
$$




\section{Approximate computation of wave-current bed shear stresses}

Consider the case of a weak current, collinear with the waves, and specified by a reference current velocity $u_{c}=u_{\text {ref }}$ at an elevation $z=z_{\text {ref }}$ above the bottom. The total bed shear stress is the sum of the current and wave contributions:

$$
\tau_{b}=\tau_{b c}+\tau_{b w}
$$

where the wave shear stress, $\tau_{b w}$, is given by equation (12), with the friction factors and phase shifts detailed above. To be consistent with having neglected the mean wave shear stress, which in the analytical model was assumed to be of the same order as the current shear stress, the approximate formulas for $\tau_{b w}$ above must be applied with $X=u_{b m, 1} /\left(k_{n} \omega\right)$, regardless of whether a current is present. Once $\tau_{b w}$ is determined, the combined wave-current shear velocity $u_{*_{w c}}$ is computed using (22) and (23). Then, $u_{*_{c}}$ is determined by matching the current velocity profile to the reference current velocity. To simplify the calculation, it is suggested that the current velocity profile used in this matching is obtained by assuming $\tau_{c} \sim \tau_{b c}=$ constant. With this assumption,

$$
u_{c} \approx u_{*_{c}}^{2} \int_{z_{0}}^{z} \frac{1}{v\left(z^{\prime}\right)} d z^{\prime}
$$

where $z_{0}=k_{n} / 30$ for rough turbulent flow, and the mean eddy viscosity is given by (4). The approximate current velocity profile is then obtained by integrating (25):

$$
u_{c}(z)= \begin{cases}\frac{u_{*_{c}}^{2}}{\kappa u_{*_{w c}}} \ln \frac{z}{z_{0}} & z_{0} \leq z \leq \delta_{I} \\ \frac{u_{*_{c}}^{2}}{\kappa u_{*_{w c}}}\left(z-\delta_{I}+\ln \frac{\delta_{I}}{z_{0}}\right) & \delta_{I}<z \leq \delta_{J} \\ \frac{u_{*_{c}}^{2}}{\kappa u_{* w c}}\left(\delta_{J}-\delta_{I}+\ln \frac{\delta_{I}}{z_{0}}\right)+\frac{u_{*_{c}}}{\kappa} \ln \frac{z}{\delta_{J}} & \delta_{J}<z \leq \delta_{K} \\ \frac{u_{*_{c}}^{2}}{\kappa u_{* w c}}\left(\delta_{J}-\delta_{I}+\ln \frac{\delta_{I}}{z_{0}}\right)+\frac{u_{*_{c}}}{\kappa}\left(z-\delta_{K}+\ln \frac{\delta_{K}}{\delta_{J}}\right) & \delta_{K}<z .\end{cases}
$$

By matching (26) with the reference current velocity, $u_{c}\left(z=z_{r e f}\right)=u_{r e f}$, the value of $u_{*_{c}}$ is determined. Finally, the current shear stress is

$$
\tau_{b c}=\rho u_{* c}^{2} .
$$

\section{BEDLOAD PREDICTIONS AND COMPARISON WITH MEASUREMENTS}

With the instantaneous bed shear stress, $\tau_{b}(t)$, obtained as detailed above, we compute bedload transport rates using Madsen's formula (Madsen 1991, 1993), same as Gonzalez-Rodriguez and Madsen (2010). The relevant equations are reproduced here for the reader's convenience; a more extensive discussion can be found in our aforementioned paper. The instantaneous bedload sediment transport rate is

$$
q_{s b}(t)=\frac{8}{(s-1) \rho g} \max \left[0,\left|\tau_{b}(t)\right|-\tau_{c r, \beta}\right] \frac{\left(\sqrt{\left|\tau_{b}(t)\right| / \rho}-\alpha_{\beta} \sqrt{\left|\tau_{c r, \beta}(t)\right| / \rho}\right)}{\cos \beta\left(\tan \phi_{m}+\tan \beta\right)} \frac{\tau_{b}(t)}{\left|\tau_{b}(t)\right|},
$$

where $\beta$ is the bottom slope in the direction of transport, taken positive if sediment is transported upslope, $s=\rho_{s} / \rho$ is the ratio between sediment and fluid densities, $\tau_{c r, \beta}$ is the critical shear stress for initiation of motion, given by

$$
\tau_{c r, \beta}=\tau_{c r, 0}\left[\cos \beta\left(1+\frac{\tan \beta}{\tan \phi_{s}}\right)\right]
$$

where $\tau_{c r, 0}$ is determined using the Shields diagram (e.g., Madsen 2001). The parameter $\alpha_{\beta}$ in (28) is given by 


$$
\alpha_{\beta}=\frac{\sqrt{\tan \phi_{m}+\tan \beta}}{\sqrt{\tan \phi_{s}+\tan \beta}},
$$

where $\phi_{\mathrm{s}} \sim 50^{\circ}$ and $\phi_{\mathrm{s}} \sim 30^{\circ}$ are the values of the angles of static and moving friction recommended by Madsen (2001).

The bedload experiments considered here correspond to sheet flow conditions, for which the effective shear stress for sediment transport, $\tau_{b}(t)$, is equal to the total bed shear stress and is thus computed using the total roughness, given by (7). Madsen's formula, (28), is only applicable as a predictor of total sediment transport rates when suspension effects are negligible. For this reason, the model can only be consistently compared to OWT experiments with $u_{*_{m}} / w_{f}<4$, where $u_{*_{m}}$ is the maximum combined (wave-current) shear velocity, and $w_{f}$ is the sediment fall velocity, calculated using Jimenez and Madsen's (2003) formula.

\section{Pure skewed waves}

Figure 1 shows a comparison between the model's predictions and experimental sediment transport data sets for purely skewed waves in oscillating water tunnels (Ribberink and Al-Salem 1994, series B, cases 7-16; Ahmed \& Sato 2003, cases U1-U13 and U15; O'Donoghue \& Wright 2004, series MA and CA; and Hassan \& Ribberink 2005, series R and Q). In the experiments, the near-bed orbital velocity is symmetric but skewed. The bed remained flat and the transport is in the sheet flow regime. All studies measured average transport rates over the entire wave cycle, with the exception of Hassan \& Ribberink's (2005) series Q, in which the onshore and offshore transport components over half-wave cycles were measured separately. The predicted bed shear stresses are based on the total mobile-bed roughness. Only bedload-dominated cases, for which the predicted $u_{* m} / w_{s}<4$, are shown in the figure. The analytical model based on the mobile-bed roughness yields good bedload predictions for skewed waves, with slight overpredictions by a factor of about 1.05 . The transport rates shown in the figure are predicted by the fitting expressions described above, and these predictions are very similar to those predicted by the exact analytical solution (reported by Gonzalez-Rodriguez and Madsen 2010).

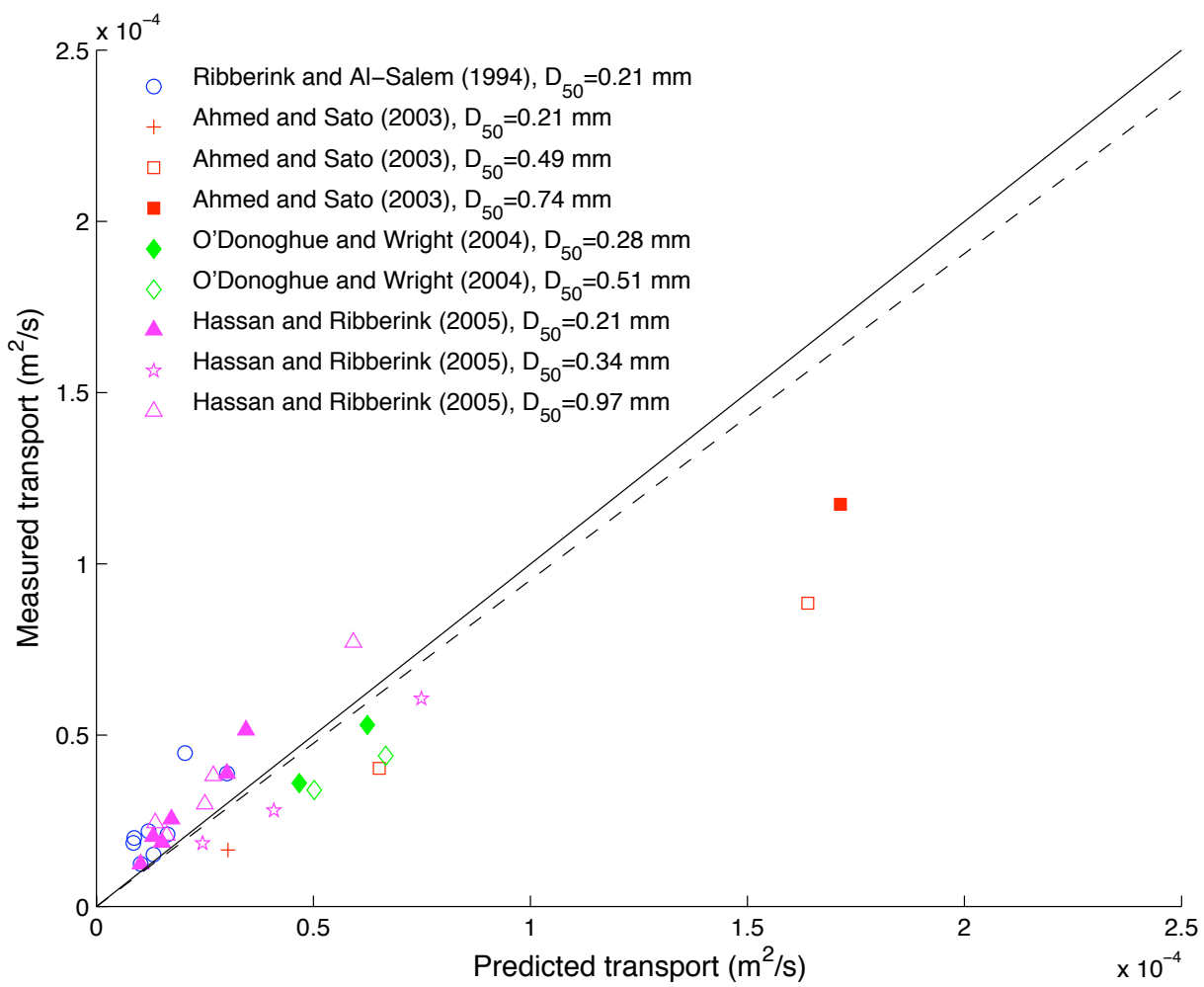

Figure 1. Comparison between measured and predicted average sediment transport rates under skewed, symmetric waves for bedload-dominated cases $\left(u_{* m} / w_{s}<4\right)$. Predictions are obtained using the approximate fittings with mobile-bed roughness. The solid line corresponds to perfect agreement between predictions and measurements, while the dashed line is the least-squares fit to the data (excluding the two data points with the largest transport rates) and corresponds to a slight overprediction by a factor of 1.05 . 


\section{Pure asymmetric waves}

Figure 2 shows a comparison between the model's predictions and experimental sediment transport data sets for purely asymmetric waves in oscillating water tunnels (King 1991, steep front and steep rear wave seriesWatanabe \& Sato 2004; and van der A et al. 2010, coarse and medium sand series). King's runs are forward- and backward-leaning half waves, consisting of a forward stroke of the wave maker. In the figure, forward-leaning waves (empty symbols) and backward-leaning waves (full symbols) result in positive and negative transports, respectively; this simply means that the net bedload transport is for all cases in the direction in which the asymmetric wave leans forward. Unlike King, Watanabe \& Sato and van der A et al. simulated the complete oscillatory motion and measured the average transport rate over the entire wave cycle under forward-leaning waves. The predicted bed shear stresses are based on the total mobile-bed roughness. Only bedload-dominated cases, for which the predicted $u_{* m} / w_{s}<4$, are shown in the figure. The predictions reasonably agree with the data, with a mean underprediction by a factor of 1.3), although there is a larger disagreement with Watanabe and Sato's coarse grain cases. The transport rates shown in the figure are predicted by the fitting expressions described above, and these predictions are close to those predicted by the exact analytical solution (reported by Gonzalez-Rodriguez and Madsen 2010).

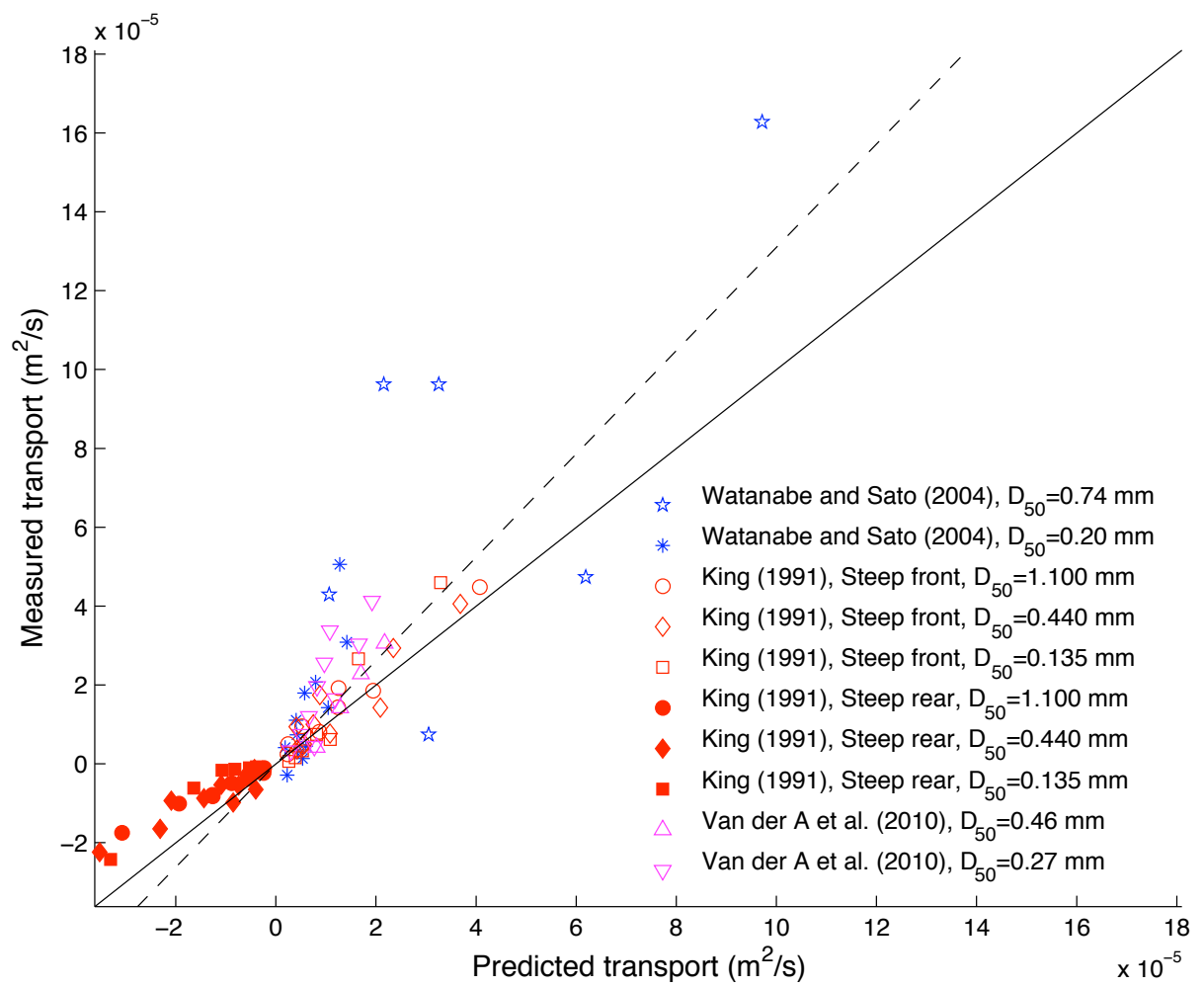

Figure 2. Comparison between measured and predicted average sediment transport rates under asymmetric, non-skewed waves for bedload-dominated cases $\left(u_{* m} / w_{s}<4\right)$. Predictions are obtained using the approximate fittings with mobile-bed roughness. The solid line corresponds to perfect agreement between predictions and measurements; the dashed line is the least-squares fit to the data (underprediction by a factor of 1.3).

\section{Sinusoidal waves plus a current}

Figure 3 shows a comparison between predictions of the analytical model based on the mobile-bed roughness and the experimental data set for sinusoidal waves plus a current by Dohmen-Janssen et al. (2002, series E, I, and J). Only bedload-dominated cases, for which the predicted $u_{* m} / w_{s}<4$, are shown in the figure, where $u_{* m}$ is now the maximum combined wave-current shear velocity. The predictions, based on the fittings and the simplified evaluation of the current stress discussed above, agree very well with the measurements, with a mean overprediction by a factor of 1.1. Unlike the cases of pure skewed and pure asymmetric waves discussed above, where the fitting expressions yield results virtually identical to the exact analytical solutions, the use of the simplified evaluation of the current shear stress here yields appreciable differences with the exact analytical expressions used by Gonzalez-Rodriguez and Madsen (2010), which result in larger overpredictions of the measurements (by a factor of about 
1.6). Such appreciable differences in the net bedload transport predictions arise however from small differences in the predicted bed shear stresses, which the net transport is highly sensitive to. For example, a maximum difference of a $4 \%$ in the instantaneous bed shear stress prediction can result in a $27 \%$ difference in the predicted net bedload transport (Gonzalez-Rodriguez 2009).

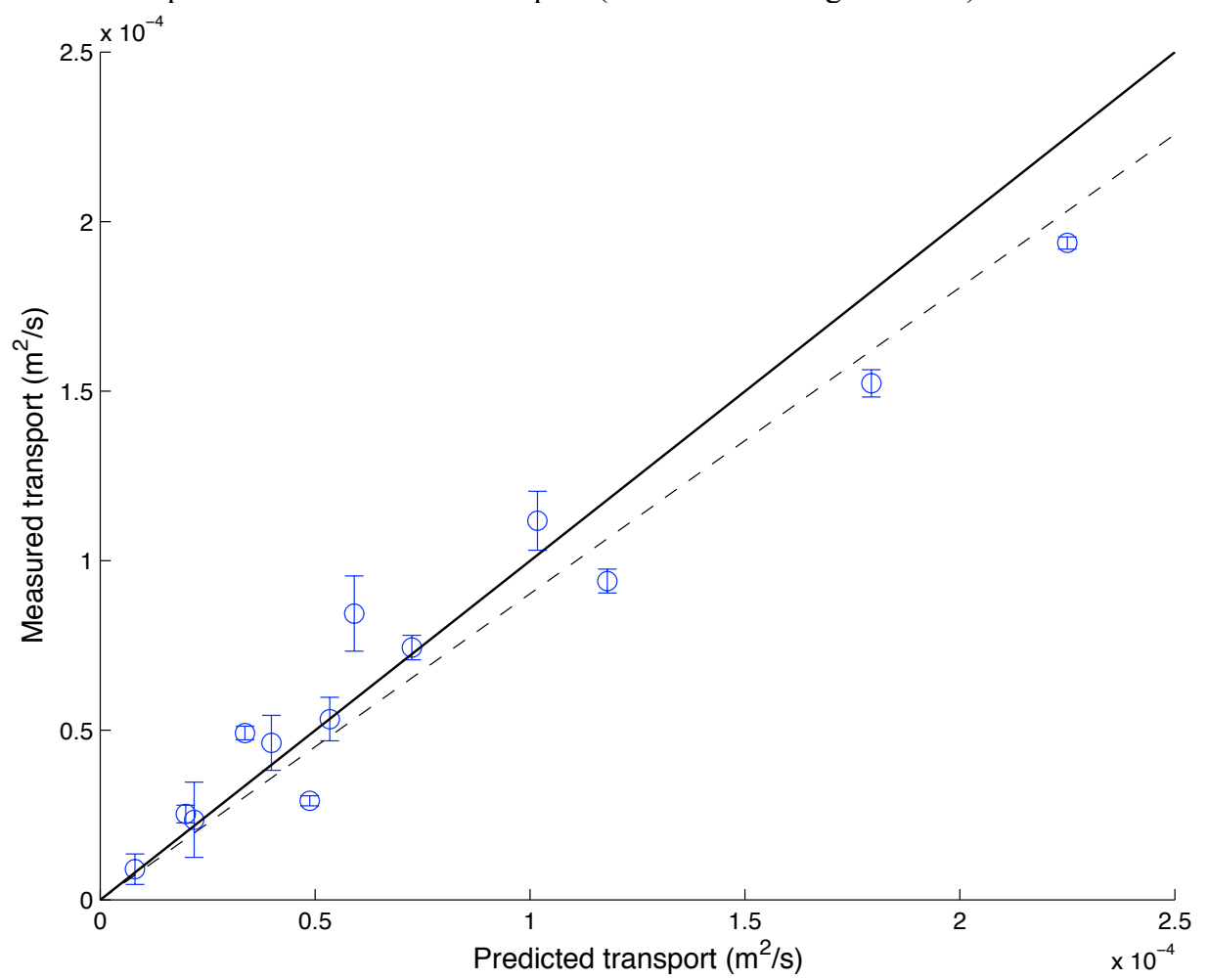

Figure 3. Comparison between measured (Dohmen-Janssen et al. 2002) and predicted average sediment transport rates in current direction for co-directional sinusoidal waves and currents for bedload-dominated cases $\left(u_{* m} / w_{s}<4\right)$. Predictions are obtained using the approximate fittings with mobile-bed roughness. The solid line corresponds to perfect agreement between predictions and measurements, while the dashed line is the least-squares fit to the data and corresponds to an overprediction by a factor of 1.1.

\section{EFFECT OF WAVE PROPAGATION}

In the previous discussion we presented a simple formulation of GRM10. The success of GRM10 to reproduce the hydrodynamics and sediment transport in OWT confirm the validity of the proposed analytical approach to study the most relevant case of propagating waves. Here we evaluate the effect of wave propagation on sediment transport; specifically, we discuss how the bedload sediment transport rates would differ between a non-propagating wave (such as in an OWT) and a propagating wave (in a wave flume or in the sea). To this end, we will use the analytical results for propagating waves obtained by Trowbridge and Madsen (1984a,b). Unlike Trowbridge and Madsen's analysis, GRM10 accounts for an imposed flux (such as a superimposed weak current) and uses a more realistic vertical structure of the eddy viscosity, which is needed to correctly model OWT streaming profiles as well as wave-current flows. However, for the case of bedload under pure waves, the second-order hydrodynamic effects associated with the streaming profile can be neglected, and a good approximation of the bed shear stress is provided by Trowbridge and Madsen's results. Indeed, as shown by GonzalezRodriguez (2009), the net bedload transport rates under pure asymmetric or skewed waves predicted using GRM10 (non-propagating waves) or Trowbridge and Madsen's (1984a,b) expressions in the limit of a very long wave only differ by about a $10-20 \%$. Thus, in this last section we use Trowbridge and Madsen's bed shear stress solutions together with Madsen's bedload formula, (28), to evaluate the effect of wave propagation on bedload transport rates under pure waves. Predictions are based on the total-mobile bed roughness given by Herrmann and Madsen's (2007) formula, (7).

Figure 4 illustrates the effect of wave propagation on net bedload transport rates. The figure shows a comparison between the predictions of Trowbridge and Madsen's solution for propagating waves and the experimental data sets of purely skewed waves in OWTs (non-propagating). The near-bed 
velocities used in Trowbridge and Madsen's model are those from the OWT experiments, but they are assumed to correspond to propagating waves in a water depth of $3 \mathrm{~m}$. The corresponding wavelengths are determined using the linear dispersion relationship for gravity waves. As shown in the figure, the transport rates predicted for propagating waves are typically 3 times larger than those measured in OWTs, demonstrating the significant effect of wave propagation on sediment transport.

Figure 5 shows a comparison between the predictions of Trowbridge and Madsen's solution for propagating waves and the experimental data sets of purely asymmetric waves in OWTs (nonpropagating). Again, predictions are obtained by assuming propagating waves in a water depth of $3 \mathrm{~m}$. The effect of wave propagation is not as clear as for the skewed waves: King's experimental cases are mostly unaffected by wave propagation, while some of Van der A et al.'s and Watanabe and Sato's data are drastically affected. Globally, however, the comparison suggests again that the measured transport rates for an asymmetric wave in an OWT may in some cases be very different from those occurring in the sea.

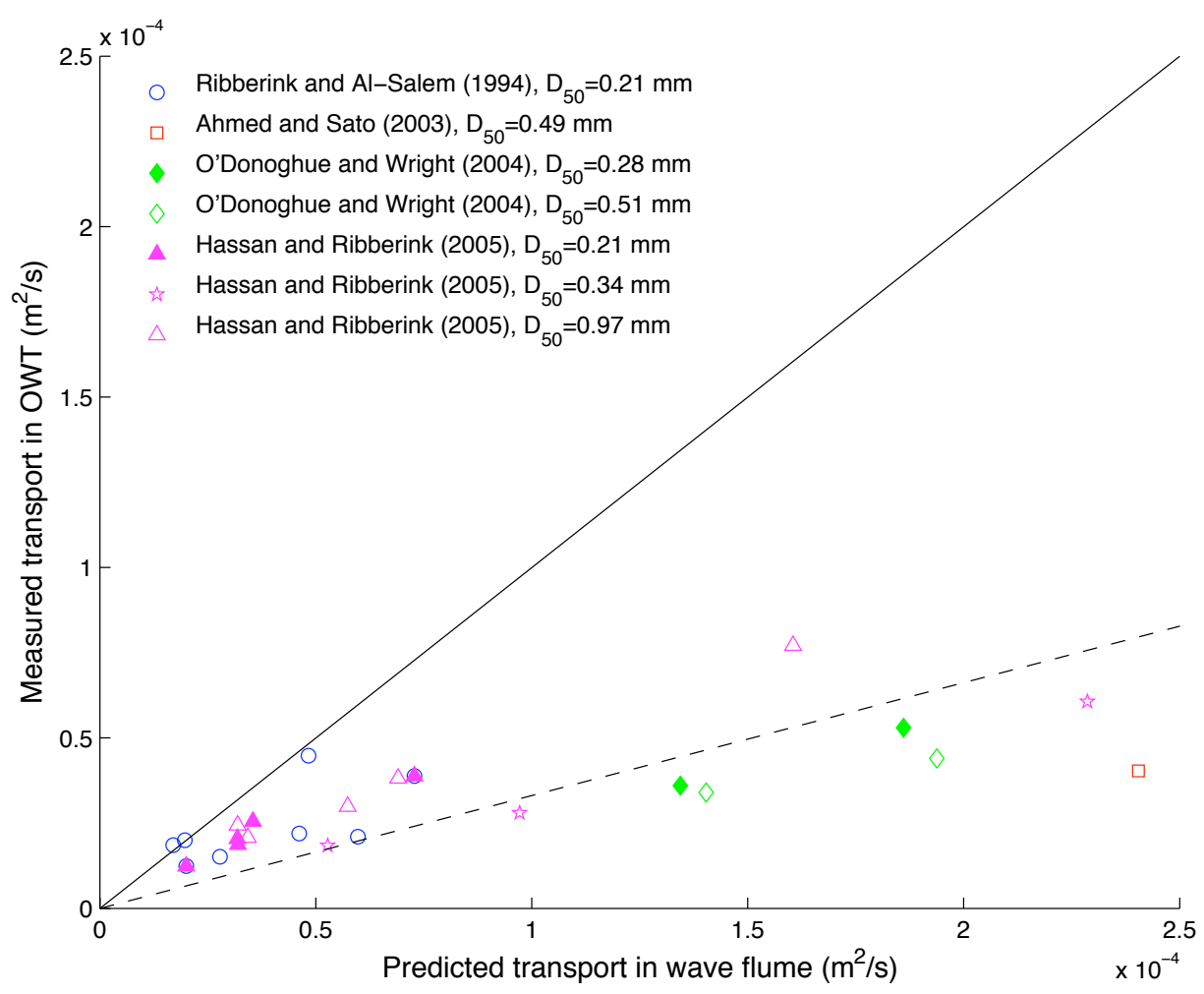

Figure 4. Comparison between measured and predicted average sediment transport rates under skewed, symmetric waves for bedload-dominated cases $\left(u_{* m} / w_{s}<4\right)$. Predictions are obtained using Trowbridge and Madsen's analytic boundary layer model with mobile-bed roughness. A water depth of $3 \mathrm{~m}$ is assumed, and the wavelength is obtained using the dispersion relationship for linear gravity waves. The solid line corresponds to perfect agreement between predictions (in wave flume conditions) and measurements (in OWTs), while the dashed line is the least-squares fit to the data and corresponds to a factor of 3.0 increase between non-propagating and propagating waves. 


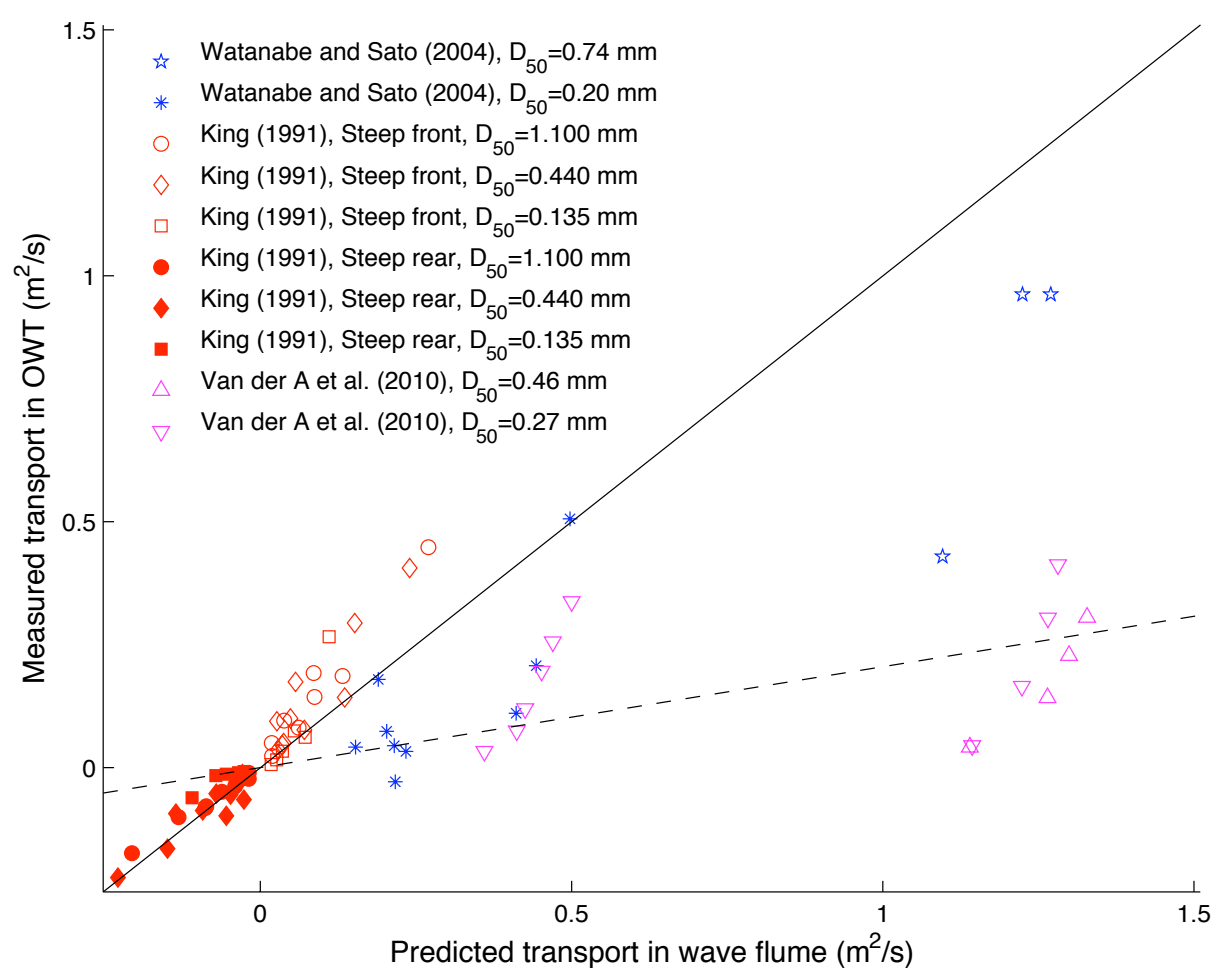

Figure 5. Comparison between measured and predicted average sediment transport rates under asymmetric, non-skewed waves for bedload-dominated cases $\left(u_{* m} / w_{s}<4\right)$. Predictions are obtained using Trowbridge and Madsen's analytic boundary layer model with mobile-bed roughness. A water depth of $3 \mathbf{m}$ is assumed, and the wavelength is obtained using the dispersion relationship for linear gravity waves. The solid line corresponds to perfect agreement between predictions (in wave flume conditions) and measurements (in OWTs), while the dashed line is the least-squares fit to the data (including three large-transport cases not shown in the figure) and corresponds to a factor of 4.9 increase between non-propagating and propagating waves.

\section{CONCLUSION}

We have presented an approximate, alternative formulation of the bed shear stress solutions of GRM10, a model of hydrodynamics and bedload transport in OWTs developed by Gonzalez-Rodriguez and Madsen (2010). The new formulation is much faster and simpler to evaluate than the original. In this approximate formulation, the first, second, and third harmonics of the bed shear stress are evaluated separately using fitted expressions for friction factors and phase shifts, and then added up to obtain the total bed shear stress. We also presented simplified expressions to compute the current shear stress in the case where an external current is prescribed through a reference current velocity. Using the approximate expressions, which (as GRM10) involve no calibration parameters, we successfully predicted bedload transport rates measured in several sheet-flow experimental studies for skewed waves, asymmetric waves, and sinusoidal waves plus a current. In all cases, good predictions were obtained when the bed shear stress is based on the total, mobile-bed roughness, evaluated using Herrmann and Madsen's (2007) formula.

We also discussed the effect of wave propagation on bedload sediment transport rates. Using the propagating-wave model of Trowbridge and Madsen (1984a,b), we predict a significant increase due to wave propagation (by a factor of 3 or more) of the bedload transport rates under pure skewed or asymmetric waves. This is an important difference that must be accounted for when validating nearshore transport models: a nearshore transport model cannot be directly validated against OWT data without accounting for the hydrodynamic differences between OWT motion and propagating waves. Accordingly, GRM10 was specifically developed for non-propagating wave conditions and consistently compared with OWT data. Therefore, we expect that an extension of the analytical approach used in GRM10 to the general case of propagating waves will be successful in predicting nearshore sediment transport processes. 


\section{ACKNOWLEDGMENTS}

The financial support of Singapore National Research Foundation (NRF) through the SingaporeMIT Alliance for Research and Technology's (SMART) Center for Environmental Sensing and Modeling (CENSAM) is gratefully acknowledged.

\section{REFERENCES}

Van der A, D.A., T. O’Donoghue, and J.S. Ribberink. 2010. Measurements of sheet flow transport in acceleration-skewed oscillatory flow and comparison with practical formulations. Coastal Engineering, 57, 331-342.

Ahmed, A.S.M., and S. Sato. 2003. A sheetflow transport model for asymmetric oscillatory flows. Part I: Uniform grain size sediments. Coastal Engineering Journal, 45, 321-337.

Dohmen-Janssen, C.M., D.F. Kroekenstoel, W.N. Hassan, and J.S. Ribberink. 2002. Phase lags in oscillatory sheet flow: experiments and bed load modeling. Coastal Engineering, 46, 61-87.

Gonzalez-Rodriguez, 2009. Wave boundary layer hydrodynamics and cross-shore sediment transport in the surf zone. PhD thesis, Massachusetts Institute of Technology, Cambridge, MA, USA.

Gonzalez-Rodriguez, D., and O.S. Madsen, 2007. Seabed shear stress and bedload transport due to asymmetric and skewed waves. Coastal Engineering, 54, 914-929.

Gonzalez-Rodriguez, D., and O.S. Madsen, 2008. Bedload transport due to asymmetric and skewed waves plus a current. Proceedings of the $31^{\text {st }}$ International Conference on Coastal Engineering, pp. 1596-1605, World Scientific.

Gonzalez-Rodriguez, D., and O.S. Madsen. 2010. Boundary layer hydrodynamics and bedload sediment transport in oscillating water tunnels. Journal of Fluid Mechanics, in press.

Hassan, W.N., and J.S. Ribberink. 2005. Transport processes of uniform and mixed sands in oscillatory sheet flow. Coastal Engineering, 52, 745-770.

Herrmann, M.J., and O.S. Madsen. 2007. Effect of stratification due to suspended sand on velocity and concentration distribution in unidirectional flows. Journal of Geophysical Research, 112, C02006.

Jimenez, J.A., and O.S. Madsen. 2003. A simple formula to estimate settling velocity of natural sediments. Journal of Waterway, Port, Coastal and Ocean Engineering, 129, 70-78.

King, D.B. 1991. Studies in Oscillatory Flow Bedload Sediment Transport. PhD thesis, University of California, San Diego.

Madsen, O.S. 1991. Mechanics of cohesionless sediment transport in coastal waters. Proceedings of Coastal Sediments '91, pp. 15-27, ASCE.

Madsen, O.S. 1993. Sediment Transport Outside the Surf Zone. Technical report. U.S. Army Engineer Waterways Experiment Station, Vicksburg, MS.

Madsen, O.S. 2001. Sediment Transport Outside the Surf Zone. In Coastal Engineering Manual, Vol. III, Chapter 6. U.S. Army Corps of Engineers, Washington DC.

O'Donoghue, T., and S. Wright. 2004. Flow tunnel measurements of velocities and sand flux in oscillatory sheet flow for well-sorted and graded sands. Coastal Engineering, 51, 1163-1184.

Ribberink, J.S., and A.A. Al-Salem. 1994. Sediment transport in oscillatory boundary layers in cases of rippled beds and sheet flow. Journal of Geophysical Research, 99, 12707-12727.

Trowbridge, J., and O.S. Madsen. 1984a. Turbulent wave boundary layers. 1. Model formulation and first-order solution. Journal of Geophysical Research, 89(C5): 7989-7997.

Trowbridge, J., and O.S. Madsen. 1984b. Turbulent wave boundary layers. 2. Second-order theory and mass transport. Journal of Geophysical Research, 89(C5): 7999-8007. 